\title{
Electrocardiographic Outcome of Resynchronization Therapy
}

\author{
O. KITTNAR ${ }^{1}$, L. RIEDLBAUCHOVÁ ${ }^{2}$, J. TOMIS $^{2}$, M. LOŽEK ${ }^{3}$, A. VALERIÁNOVÁ ${ }^{1}$, \\ M. HRACHOVINA ${ }^{1}$, M. MLČEK ${ }^{1}$, M. HUPTYCH ${ }^{4}$, J. JANOUŠEK ${ }^{3}$, L. LHOTSKÁ ${ }^{4}$
}

${ }^{1}$ Institute of Physiology, First Faculty of Medicine, Charles University in Prague, Czech Republic, ${ }^{2}$ Department of Cardiology, University Hospital in Motol and Second Faculty of Medicine, Charles University in Prague, Czech Republic, ${ }^{3}$ Children's Heart Centre, Motol Children's Hospital, Prague, Czech Republic, ${ }^{4}$ Czech Institute of Informatics, Robotics, and Cybernetics, Czech Technical University, Prague, Czech Republic

Received March 24, 2017

Accepted November 8, 2017

\section{Summary}

Cardiac resynchronization therapy (CRT) has proven efficacious in reducing or even eliminating cardiac dyssynchrony and thus improving heart failure symptoms. However, quantification of mechanical dyssynchrony is still difficult and identification of CRT candidates is currently based just on the morphology and width of the QRS complex. As standard 12-lead ECG brings only limited information about the pattern of ventricular activation, we aimed to study changes produced by different pacing modes on the body surface potential maps (BSPM). Total of 12 CRT recipients with symptomatic heart failure (NYHA II-IV), sinus rhythm and QRS width $\geq 120$ ms and 12 healthy controls were studied. Mapping system Biosemi (123 unipolar electrodes) was used for BSPM acquisition. Maximum QRS duration, longest and shortest activation times (ATmax and ATmin) and dispersion of QT interval (QTd) were measured and/or calculated during spontaneous rhythm, single-site right- and left-ventricular pacing and biventricular pacing with ECHO-optimized AV delay. Moreover we studied the impact of CRT on the locations of the early and late activated regions of the heart. The average values during the spontaneous rhythm in the group of patients with dyssynchrony (QRS $140.5 \pm 10.6 \mathrm{~ms}$, ATmax $128.1 \pm 10.1 \mathrm{ms,}$ ATmin $31.8 \pm 6.7 \mathrm{~ms}$ and QTd $104.3 \pm 24.7 \mathrm{~ms}$ ) significantly differed from those measured in the control group (QRS 93.0 $\pm 10.0 \mathrm{~ms}$, ATmax $79.1 \pm 3.2 \mathrm{~ms}$, ATmin $24.4 \pm 1.6 \mathrm{~ms}$ and QTd 43.6 $\pm 10.7 \mathrm{~ms}$ ). Right ventricular pacing (RVP) improved significantly only ATmax $[111.2 \pm 10.6 \mathrm{~ms}(\mathrm{p}<0.05)]$ but no other measured parameters. Left ventricular pacing (LVP) succeeded in improvement of all parameters [QRS $105.1 \pm 8.0 \mathrm{~ms}(p<0.01)$, ATmax 103.7 $\pm 7.1 \mathrm{~ms}(p<0.01)$, ATmin $20.2 \pm 3.7 \mathrm{~ms}(p<0.01)$ and QTd 52.0 $\pm 9.4 \mathrm{~ms}(\mathrm{p}<0.01)]$. Biventricular pacing (BVP) showed also a beneficial effect in all parameters [QRS $121.3 \pm 8.9 \mathrm{~ms}(p<0.05)$, ATmax $114.3 \pm 8.2 \mathrm{~ms}(p<0.05)$, ATmin $22.0 \pm 4.1 \mathrm{~ms}(p<0.01)$ and QTd $49.8 \pm 10.0 \mathrm{~ms}(p<0.01)]$. Our results proved beneficial outcome of LVP and BVP in evaluated parameters (what seems to be important particularly in the case of activation times) and revealed a complete return of activation times to normal distribution when using these CRT modalities.

\section{Key words}

Cardiac resynchronization therapy • Heart failure • Dyssynchrony

- Electrocardiography - Body surface mapping - Biventricular pacing

\section{Corresponding author}

O. Kittnar, Institute of Physiology, First Faculty of Medicine Charles University in Prague, Albertov 5, 12800 Prague 2, Czech Republic. E-mail: otomar.kittnar@lf1.cuni.cz

\section{Introduction}

Healthy myocardium is activated by high-velocity depolarization waveform that propagates through the conducting system of the heart, resulting in synchronized depolarization of the ventricles. The heart failure (HF) is known to be associated with electrical and conduction abnormalities. Altered velocity and uniformity of depolarization waveform propagation causes delayed electrical activation of some ventricular areas. The product of significantly impaired conduction velocity can be electrical dyssynchrony of the ventricles manifesting as lengthening of the QRS complex and 
change of its morphology on the surface electrocardiogram. Due to electromechanical coupling, delayed ventricular electrical activation (typically of left bundle branch block morphology) is often associated with corresponding mechanical dyssynchrony. One consequence of long-standing cardiac dyssynchrony is a pathological process known as myocardial remodeling which manifests clinically as left ventricle (LV) dilatation, worsening of the LV systolic and diastolic function, and progressive HF (Jaffe and Morin 2014). Importantly, it was shown that a direct relationship exists between QRS duration and reduced LV ejection fraction (Shenkman et al. 2002).

Pacemaker-based treatment termed cardiac resynchronization therapy (CRT) was developed to restore more coordinated activation pattern of the heart. Despite modifying primarily electrical dyssynchrony, CRT is able to correct mechanical asynchrony as well, thus improving heart failure symptoms and inducing reverse remodeling leading to reduced mortality and hospitalization rate in patients with advanced $\mathrm{HF}$.

Although the goal of CRT is to ensure more coordinated contraction pattern, our current possibilities to detect and quantify mechanical dyssynchrony in CRT candidates are still only limited. Therefore we presume that presence and degree of mechanical dyssynchrony correspond with the severity of electrical asynchrony that is reflected in the altered morphology and duration of the QRS complex which can be easily detectable on a surface ECG. However, the QRS width reflects rather a total duration of ventricular activation and doesn't provide information about the space and temporal characteristics of the ventricular activation pattern (Fauchier et al. 2002). With regard to QRS morphology, CRT is very effective in patients with left bundle-branch block (LBBB) pattern where the region of late activation (although being interindividually variable) is usually located in the basal segments of the LV lateral wall. These segments are highly overloaded as the contractile dyssynchrony resulting from electrical dyssynchrony generates marked regional heterogeneity of myocardial work, with the early stimulated region having reduced load and territories of late activation exhibiting higher load (Kirk and Kass 2013). However, standard 12-lead ECG brings only limited information about the pattern of ventricular activation. Therefore, we decided to study changes produced by different pacing modes on the body surface potential maps (BSPM) as a noninvasive tool that brings more detail information with this regard. Accordingly, the aims of the present study were: 1) to evaluate the effect of CRT on surface electrocardiographic findings and 2) to study the impact of CRT on the locations of the early and late activated regions of the heart during BSPM.

\section{Methods and pacients}

Electrocardiographic recordings were obtained from 12 patients ( 7 women, 5 men, age $61.5 \pm 6.1$ years) who underwent implantation of CRT pacemaker/ defibrillator in accordance with current guidelines (2013 ESC Guidelines 2013), i.e. symptomatic HF in New York Heart Association (NYHA) functional class II to IV despite adequate medical treatment, left ventricular ejection fraction $\leq 35 \%$, sinus rhythm, PR duration $\geq 150 \mathrm{~ms}$, intraventricular conduction disturbance with QRS duration $\geq 120 \mathrm{~ms}$ in LBBB type or $\geq 150 \mathrm{~ms}$ in nonLBBB type (all $12 \mathrm{CRT}$ recipients in our study presented with LBBB, however). Three modalities of CRT pacing with a sensed AV delay between 100-120 ms were used: biventricular pacing, single left ventricular pacing and single right ventricular pacing. 8 patients $(67 \%)$ were responders, 4 patients (33\%) were non-responders.

To compare findings in CRT recipients with those observed in normal healthy subject, the same electrocardiographic recordings were obtained during spontaneous rhythm from the control group containing 12 healthy volunteers (6 women, 6 men, age $48.9 \pm 12.3$ ). A healthy person was defined for the purposes of this project according to the following findings and data: a negative cardiologic family and personal history, a normal arterial blood pressure, normal glycaemia, cholesterolaemia, ECG, non-smoker, normal body weight, a negative neurological, psychiatric and endocrinological personal history and no cardioactive medication.

The examination was performed using standard conditions; electrocardiographic and body surface potential mapping (BSPM) recordings were obtained simultaneously using the BioSemi device. Two minutes (8-12 beats) were recorded during spontaneous rhythm in both CRT recipients and healthy controls. In addition, ECG and BSPM were recorded in three different pacing modes in the CRT group after appropriate reprogramming of the device: simultaneous biventricular pacing (BVP), single-site left-ventricular pacing (LVP) and single-site right-ventricular pacing (RVP) with the same AV delay. 
Following parameters were evaluated: the longest QRS complex duration (QRS) detected in any BSPM lead, the shortest and the longest activation time (ATmin and ATmax), dispersion of the QT interval (QTd) and locations of the ATmin and ATmax. Activation time (AT) was measured between the beginning of ventricular depolarization and the $\mathrm{R}$ wave in the individual chest leads. ATmax is the longest and ATmin the shortest AT interval detected in any BSPM lead. For evaluating the QTd, the QT interval was measured by 123 unipolar chest leads used for BSPM. The QT interval was measured from the start of the Q wave to the end of the T wave, QTd was then defined as the difference between the maximal and minimal QT interval in any of the leads measured.

The parameters were labeled manually using a graphic system developed for this purpose in Matlab 8.0 programming environment. A section of the recording with constant R-R intervals and morphology was selected, from which the annotating electrophysiologist chose one beat. Parameters for all the leads were labeled from this one beat. Before labelling, signals were preprocessed using standard filtration $(0.67 \mathrm{~Hz}$ highpass and $45 \mathrm{~Hz}$ lowpass FIR filters, $50 \mathrm{~Hz}$ notch filter and DC offset removal).

Statistical comparison was performed using the nonparametric Mann-Whitney test.

\section{Results}

The findings are summarized in Figure 1. The average values of all parameters during the spontaneous rhythm significantly differed in the CRT group (QRS 140.5 $\pm 10.6 \mathrm{ms,} \mathrm{ATmax} 128.1 \pm 10.1 \mathrm{~ms}$, ATmin $31.8 \pm 6.7 \mathrm{~ms}$ and QTd $104.3 \pm 24.7 \mathrm{~ms}$ ) from those measured in the control group (QRS 93.0 $\pm 10.0 \mathrm{~ms}$, ATmax $79.1 \pm 3.2 \mathrm{~ms}$, ATmin $24.4 \pm 1.6 \mathrm{~ms}$ and QTd 43.6 $\pm 10.7 \mathrm{~ms}$ ). Right ventricular pacing (RVP) improved significantly only ATmax $[1112 \pm 10.6 \mathrm{~ms}$ $(p<0.05)]$ but no other measured parameter. Left ventricular pacing (LVP) succeeded in improvement of all parameters $[\mathrm{QRS} 105.1 \pm 8.0 \mathrm{~ms}(\mathrm{p}<0.01)$, ATmax $103.7 \pm 7.1 \mathrm{~ms}(\mathrm{p}<0.01)$, ATmin $20.2 \pm 3.7 \mathrm{~ms}(\mathrm{p}<0.01)$ and QTd $52.0 \pm 9.4 \mathrm{~ms} \quad(\mathrm{p}<0.01)]$ as compared to the spontaneous rhythm of CRT patients. Biventricular pacing (BVP) showed also a beneficial effect in all parameters [QRS $121.3 \pm 8.9 \mathrm{~ms} \quad(\mathrm{p}<0.05), \quad$ ATmax $114.3 \pm 8.2 \mathrm{~ms}(\mathrm{p}<0.05)$, ATmin $220 \pm 4.1 \mathrm{~ms}(\mathrm{p}<0.01)$ and QTd $49.8 \pm 10.0 \mathrm{~ms}(\mathrm{p}<0.01)]$, however degree of their improvement was slightly worse as compared to LVP. Although neither BVP nor LVP induced normalization of QRS duration and ATmax as compared to normal healthy subjects, both BVP and LVP exhibited their significant shortening to values observed in the control group.

Regarding the locations of the early and late activated regions of the heart, both LVP and particularly BVP were associated with their distribution that resembled findings in healthy controls. The effect of BVP is summarized in Figure 2. In HF patients the late activation site (ATmax) during spontaneous rhythm was located in a left upper precordium (representing the anterobasal portion of the left ventricular surface) and after LVP or BVP stimulation it shifted to a lower back part of the chest (i.e. to a surface of the posterior wall). The location of the early activation (ATmin) during spontaneous rhythm was in a right lower back part of the chest in the CRT group (i.e. a surface of the right ventricular wall) and by pacing it shifted to the left lower precordium (i.e. a surface of the anterior wall close to the apex). The RVP was not able to shift the early and late activated regions to the normal distribution.

\section{Discussion}

The present study demonstrated that in HF patients with electrical dyssynchrony, CRT may induce significant improvement of the tested parameters. However, one must be cautious with extrapolation that observed electrical resynchronization led to similar mechanical resynchronization. It applies in particular to the shortening of the QRS complex. Although the duration of QRS complex is used as a measure of particularly left ventricular dyssynchrony to select patients for CRT (Dickstein et al. 2010), it was shown poor correlation between the QRS width and the mechanical dyssynchrony exists (Auger et al. 2012, Bleeker et al. 2004). With regard to morphology of the QRS complex, Zareba et al. (2011) have shown that the patients with LBBB exhibit a greater clinical benefit from CRT compared with patients with other QRS complex morphologies. Since there were no nonLBBB patients included in our study so far, we could not compare how patients with $\mathrm{LBBB}$ versus nonLBBB pattern differ in the tested parameters.

Clearer is the interpretation of activation time, particularly the ATmax. Sweeney et al. (2010) showed that patients with shorter LV activation time delays ( $\leq 80 \mathrm{~ms}$ ) had a $51 \%$ response rate to CRT compared 
A

QRS (ms)
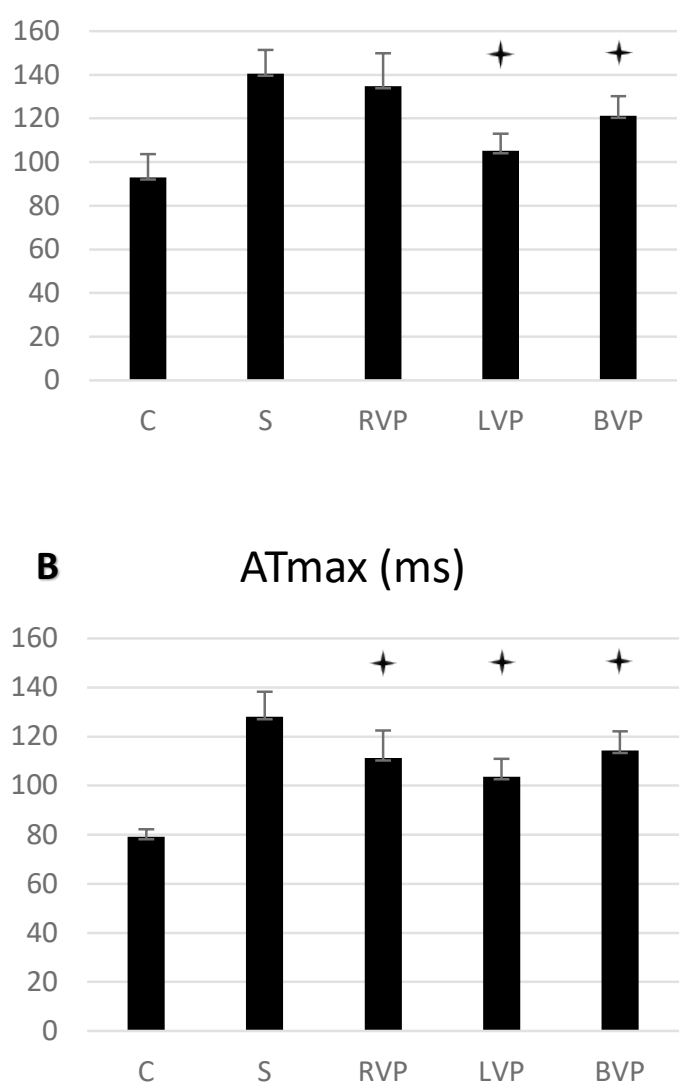

C

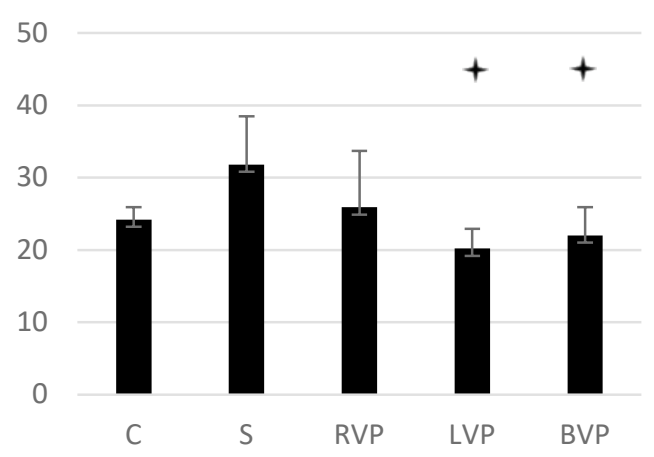

D

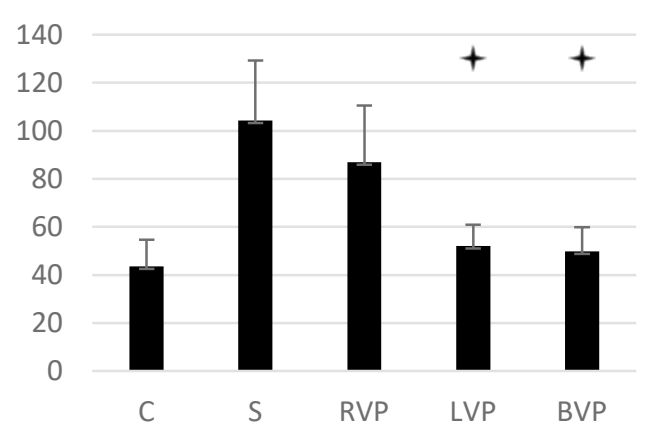

Fig. 1. Graphs of the results. (A) width of the QRS complex (QRS), (B) the longest activation time (ATmax), (C) the shortest activation time (ATmin), (D) dispersion of the QT interval (QTd). C - spontaneous rhythm in control group (healthy subjects), $\mathrm{S}$ - spontaneous rhythm in CRT recipients; pacing modes tested in CRT recipients (significant differences from $\mathrm{S}$ are labeled by stars): RVP - right ventricular pacing, LVP - left ventricular pacing, BVP - biventricular pacing.

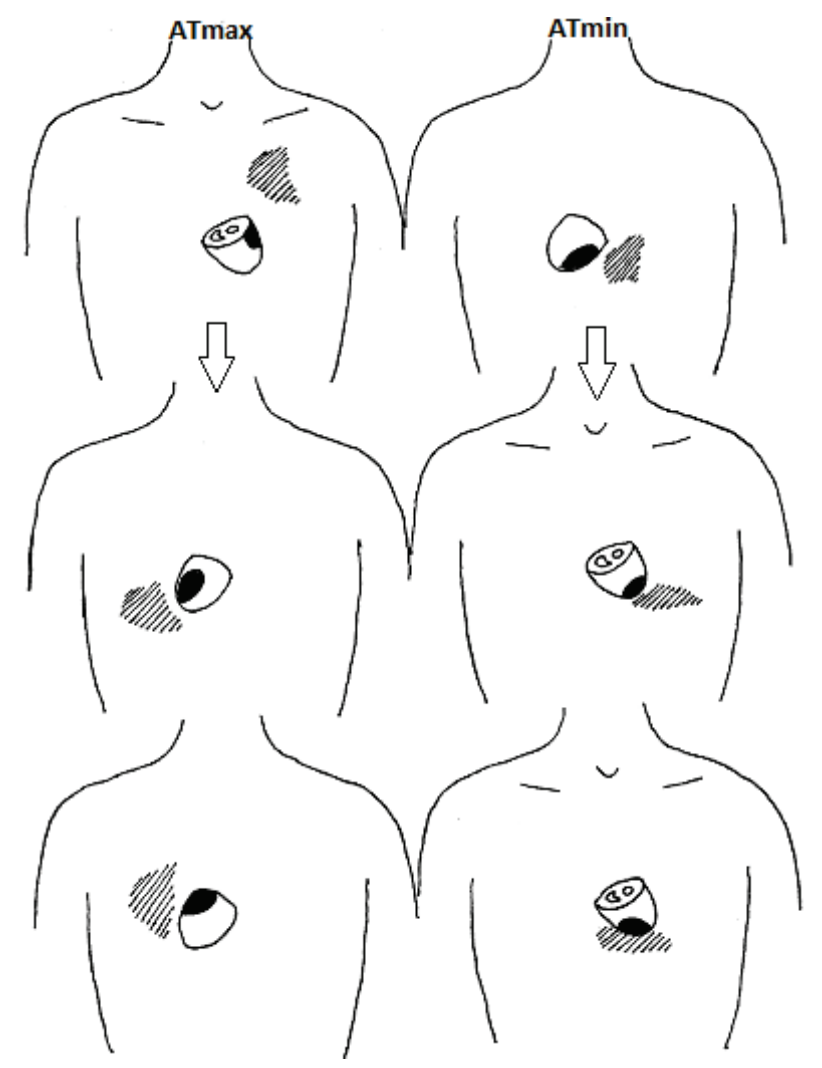

Fig. 2. Distribution of the longest activation times during spontaneous rhythm in CRT recipients (left upper scheme) and its shift after CRT (left middle scheme). Distribution of the shortest activation times during spontaneous rhythm (right upper scheme) in CRT recipients and its shift after CRT (right middle scheme). Lower schemes represent distribution of the longest activation times (left) and the shortest activation times (right) in healthy controls. Dashed area on the chest surface comprises locations where the activation times were detected, black area on the ventricles surface represents the locations of activation times that correspond to the detected areas on the body surface. Front chest surface is on the left, the back one is on the right.

with $73 \%$ response in patients with larger LV activation time delays $(\geq 125 \mathrm{~ms})$. These differences in LV activation time delays may result in different LV mechanical activation patterns that determine a different response to CRT (Auger et al. 2012). Therefore, it seems that ATmax could be better marker of the CRT effect than the QRS duration although this hypothesis has to be tested in a larger study yet. Similarly, the location of the region with activation time 
delay could be interesting from this point of view. Moreover, a big advantage of activation time measurement is that this parameter is not sex-related. On the other hand its crucial disadvantage is that it depends on the age: it was proved that activation times are longer in older hearts (Waldeyer et al. 2009).

More complicated is the question of QTd predictive value. Our decision to use a greater number of leads for the determination of the QTd helps to determine it more accurately than an assessment with only 12 or even 6 precordial leads. The use of a low number of leads was undoubtedly the main cause of the repeatedly suggested poor reproducibility (Kautzner et al. 1994). Measurements performed in the present study indicate that electrical dyssynchrony can cause an increase in the QTd. These findings are in agreement with those from our previous studies (Lechmanova et al. 2002, Kittnar et al. 2004), from which we concluded that QTd can reflect not only an increased risk of serious arrhythmias, but that the increased QTd is just a non-specific sign of a changed course of repolarization. Thus it could be concluded that this parameter can be used just as a measure of an effect of any intervention on the myocardium, what is undoubtedly the case of the present study.

CRT represents a major advance in HF therapy. Contemporarily, it is the only clinical treatment that can both acutely and chronically modify electrical activation pattern of the ventricles thus changing systolic function (Kirk and Kass 2013). Better effect of LVP and particularly of BVP in comparison to RVP is not very surprising (Jaffe and Morin 2014). However, LVP and BVP show similarly favorable outcome. Although our study deals only with immediate effect of different pacing modalities and does not reflect long-term impact of BVP and LVP, we have a strong evidence that LVP provides positive and comparable effect as BVP. This was demonstrated in the original acute hemodynamic studies, but also during longer-term follow-up. Both subjective and objective improvement may be visible as early as in the first 3 months of BVP or LVP. Both pacing strategies are also capable to induce reverse remodeling. It is believed (and data from electroanatomical mapping supports these conclusions) that similar effect of LVP and BVP can be attributed to the fusion between LV pacinginduced activation wavefront and conduction over the native conduction system. Although LVP seems to be comparable to BVP, most of the CRT recipients are simultaneously at higher risk of sudden cardiac death. Thus implantation of defibrillation RV lead is usually performed due to this indication in most patients and BVP mode of pacing is thus used preferably in clinical practice.

The locations of the early and late activated regions of the heart after BVP stimulation could be a little bit surprising particularly in comparison with usual CARTO findings. From that point of view it is necessary to emphasize that our results are derived from a complex electrical heart field that is more complicated than endocardial distribution of electrical potentials illustrated by the CARTO method.

\section{Conflict of Interest}

There is no conflict of interest.

\section{Acknowledgements}

Supported by Ministry of Health of the Czech Republic, grant Nr. 15-31398A and SVV260379. All rights reserved.

\section{Conclusions}

Our results demonstrated improvement of evaluated parameters during LVP and BVP that mimicked findings observed in control healthy subjects. This observation seems to be important particularly in the case of activation times where it revealed a return of activation times close to normal distribution when using these CRT modalities.

\section{References}

2013 ESC Guidelines on cardiac pacing and cardiac resynchronization therapy. Eur Heart J 34: 2281-2329, 2013.

AUGER D, BLEEKER GB, BERTINI M, EWE SH, VAN BOMMEL RJ, WITKOWSKI TG, NG AC, VAN ERVEN L, SCHALIJ MJ, BAX JJ, DELGADO V: Effect of cardiac resynchronization therapy in patients without left ventricular dyssynchrony. Eur Heart J 33: 913-920, 2012.

BLEEKER GB, SCHALIJ MJ, MOLHOEK SG, VERWEY HF, HOLMAN ER, BOERSMA E, STEENDIJK P, VAN DER WALL EE, BAX JJ: Relationship between QRS duration and left ventricular dyssynchrony in patients with end-stage heart failure. J Cardiovasc Electrophysiol 15: 544-549, 2004. 
DICKSTEIN K, VARDAS PE, AURICCHIO A, DAUBERT JC, LINDE C, MCMURRAY J, PONIKOWSKI P, PRIORI SG, SUTTON R, VAN VELDHUISEN DJ, ET AL.: 2010 Focused Update of ESC Guidelines on device therapy in heart failure: an update of the 2008 ESC Guidelines for the diagnosis and treatment of acute and chronic heart failure and the 2007 ESC guidelines for cardiac and resynchronization therapy. Developed with the special contribution of the Heart Failure Association and the European Heart Rhythm Association. Eur Heart J 31: 2677-2687, 2010.

FAUCHIER L, MARIE O, CASSET-SENON D, BABUTY D, COSNAY P, FAUCHIER JP: Interventricular and intraventricular dyssynchrony in idiopathic dilated cardiomyopathy: a prognostic study with Fourier phase analysis of radionuclide scintigraphy. J Am Coll Cardiol 40: 2022-2030, 2002.

JAFFE LM, MORIN DP: Cardiac resynchronization therapy: history, present status, and future directions. Ochsner J 14: 596-607, 2014.

KAUTZNER J, GANG YI, CAMM AJ, MALIK M: Short- and Long-term reproducibility of QT, QTc and QT dispersion measurement in healthy subjects. PACE 17: 928-937, 1994.

KIRK JA, KASS DA: Electromechanical dyssynchrony and resynchronization of the failing heart. Circ Res 113: 765-776, 2013.

KITTNAR O, PACLT I, MLCEK M, SLAVÍCEK J, DOHNALOVÁ A, HAVRÁNEK S, BRIZMAN E, KITZLEROVÁ E, PISVEJCOVÁ K: QT dispersion and electrical heart field morphology in patients treated with dosulepin. Physiol Res 53: 379-386, 2004.

LECHMANOVÁ M, KITTNAR O, MLČEK M, SLAVÍČEK J, DOHNALOVÁ A, HAVRÁNEK Š, KOLǍ̌íK M, PAŘİZK A: QT Dispersion and T-Loop morphology in late pregnancy and after delivery. Physiol Res 51: 121-129, 2002.

SHENKMAN HJ, PAMPATI V, KHANDELWAL AK, MCKINNON J, NORI D, KAATZ S, SANDBERG KR, MCCULLOUGH PA: Congestive heart failure and QRS duration: establishing prognosis study. Chest 122: 528-534, 2002.

SWEENEY MO, VAN BOMMEL RJ, SCHALIJ MJ, BORLEFFS CJ, HELLKAMP AS, BAX JJ: Analysis of ventricular activation using surface electrocardiography to predict left ventricular reverse volumetric remodeling during cardiac resynchronization therapy. Circulation 121: 626-634, 2010.

WALDEYER C, FABRITZ L, FORTMUELLER L, GERSS J, DAMKE D, BLANA A, LAAKMANN S, KREIENKAMP N, VOLKERY D, BREITHARDT G, KIRCHHOF P: Regional, age-dependent, and genotype-dependent differences in ventricular action potential duration and activation time in 410 Langendorff-perfused mouse hearts. Basic Res Cardiol 104: 523-533, 2009.

ZAREBA W, KLEIN H, CYGANKIEWICZ I, HALL WJ, MCNITT S, BROWN M, CANNOM D, DAUBERT JP, ELDAR M, GOLD MR, ET AL.: Effectiveness of cardiac resynchronization therapy by QRS morphology in the Multicenter Automatic Defibrillator Implantation Trial-Cardiac Resynchronization Therapy (MADITCRT). Circulation 123: 1061-1072, 2011. 